\title{
Envelhecimento, voz e atividade física de professores e não professores
}

\author{
Aging, voice and physical activity of teachers and non-teachers
}

\author{
Deborah Gampel ${ }^{1}$, Ursula Margarida Karsch², Léslie Piccolotto Ferreira ${ }^{3}$
}

\begin{abstract}
RESUMO
Objetivo: Comparar a voz de sujeitos idosos professores e não professores e relacionar com idade cronológica e tempo de prática de atividade física. Métodos: Foi realizada a análise perceptivo-auditiva dos parâmetros vocais e o cruzamento estatístico com idade cronológica e tempo de prática de atividade física de 47 sujeitos, homens e mulheres, com idades acima de 65 anos, sendo 23 professores (GP) e 24 não professores (GNP). Resultados: Para o GP foi encontrado resultado significante entre idade cronológica e variação de loudness (correlação negativa e p=0,042), e para o GNP entre idade cronológica e velocidade (correlação negativa e p=0,038) e entre o tempo de prática de atividade física e os desvios de qualidade vocal (correlação negativa e $\mathrm{p}=0,031$ ). Conclusões: Não foi encontrada diferença estatisticamente significante entre os parâmetros vocais dos respectivos grupos. Para o GP, verificou-se que quanto maior a idade cronológica, menor a variação de loudness, e para o GNP, quanto maior a idade cronológica, menor a velocidade de fala. O maior tempo de prática de atividade física relacionou-se a uma qualidade vocal com menos desvios, apenas para GNP.
\end{abstract}

Descritores: Envelhecimento; Voz; Idoso; Docentes; Atividade motora; Exercício; Saúde do idoso

\section{INTRODUÇÃO}

O presente estudo partiu da necessidade de entender a diversidade do envelhecimento vocal, em decorrência do significativo aumento da demanda de pacientes idosos em busca de atendimento fonoaudiológico, com interesse em aprimorar a voz.

O envelhecimento é um processo universal, resultante da interação dos aspectos biológicos, funcionais, psicológicos e sociais ${ }^{(1-2)}$. Os órgãos, sistemas e funções não envelhecem ao mesmo tempo, em parte, devido à própria biologia, mas também dependem do momento histórico, das culturas, das classes sociais, das histórias pessoais de vida, das condições educacionais, dos estilos de vida, gênero, profissão e etnia. $\mathrm{O}$ modo pelo qual cada um envelhece depende de como vive, da faixa etária e da geração a que pertence; dos fatores genéticos,

Trabalho desenvolvido no Programa de Estudos Pós Graduados em Gerontologia da Pontifícia Universidade Católica de São Paulo - PUC-SP - São Paulo (SP), Brasil.

(1) Mestre Aperfeiçoamento em Distúrbios da Comunicação pela Pontifícia Universidade Católica de São Paulo - PUC-SP - São Paulo (SP), Brasil; Fonoaudióloga clínica - São Paulo (SP), Brasil.

(2) Doutora, Professora do Programa de Estudos Pós Graduados em Gerontologia da Pontifícia Universidade Católica de São Paulo - PUC-SP - São Paulo (SP), Brasil.

(3) Doutora, Professora Titular do Departamento de Fundamentos da Fonoaudiologia da Pontifícia Universidade Católica de São Paulo - PUC-SP - São Paulo (SP), Brasil; Coordenadora e docente do Curso de Especialização em Fonoaoudiologia - Voz da Pontifícia Universidade Católica de São Paulo PUC-SP - São Paulo (SP), Brasil.

Endereço para correspondência: Deborah Gampel. Al. Lorena, 1304, cj. 1106, São Paulo - SP, CEP 01424-001. E-mail: dgtichauer@attglobal.net Recebido em: 21/8/2007; Aceito em: 19/5/2008 da incidência de diferentes patologias durante o processo de desenvolvimento, e do ambiente ecológico ${ }^{(1,3)}$.

O envelhecimento, portanto, não é uniforme, pois tem variações intra e inter sujeitos, de modo que não há uma idade específica para se tornar velho ${ }^{(1)}$. O critério cronológico é adotado nos trabalhos científicos devido à dificuldade de se definir idade biológica, pois as mudanças decorrentes do processo de envelhecimento, embora claras, não são exclusivamente dependentes do envelhecimento primário e podem ser resultantes de outros fatores, tais como: sociais, psicológicos contexto socioeconômico e história de vida $^{(1-2)}$.

Apesar da grande variação individual, tanto na manifestação dos sinais físicos do envelhecimento, como na forma de adaptação psicológica de cada um a este processo, que mais cedo ou mais tarde atinge a todos, as mudanças produzidas com o avanço do tempo também atingem a laringe e os sistemas relacionados à fonação. A diminuição do controle neuromuscular, das habilidades sensoriais e dos níveis de energia reflete nos padrões vocais ${ }^{(4)}$.

Além disso, segundo a mesma autora, a presença de doenças, os efeitos de medicamentos e o estado psicológico também podem limitar as habilidades de comunicação. Embora as mudanças vocais do idoso possam ser decorrentes de doenças associadas ao envelhecimento, há alguns sinais tipicamente resultantes da idade do mecanismo vocal.

A heterogeneidade do envelhecimento, portanto, também é refletida na voz, principal ferramenta de comunicação oral do homem. Os parâmetros vocais mudam com o avanço do tempo, mas não há um consenso na literatura sobre o início, o tipo e o grau de mudança ${ }^{(4-6)}$. As principais mudanças vocais associadas ao envelhecimento são: 
- Em relação ao pitch: Para homens, há uma tendência para pitch mais agudo, enquanto que para mulheres pode haver um abaixamento do pitch, provavelmente associado ao edema pós-menopausa e à queda hormonal ${ }^{(4-5)}$.

- Quanto ao loudness: Há autores que constatam uma tendência à redução ${ }^{(4)}$, enquanto outros mencionam não haver diferença significativa quanto ao volume para conversação entre grupos de mulheres jovens ( 25 a 30 anos) e outro de idosas acima de $75 \operatorname{anos}^{(7)}$.

- Em relação à ressonância: Nas mulheres, pode ocorrer um foco de ressonância mais baixo ${ }^{(4,8)}$, enquanto que nos homens, poderia haver um aumento do grau de nasalidade $^{(5)}$.

- Quanto à variação de pitch: Pode estar diminuída ${ }^{(4,9)}$, ou pode haver um aumento na variabilidade do pitch em sujeitos idosos, independente do sexo, conforme verificado na literatura consultada ${ }^{(5)}$.

- Quanto à variação de loudness: Também foi observada uma redução ${ }^{(4,9)}$.

- Em relação à velocidade de fala: Pode ocorrer uma lentificação ${ }^{(5,10)}$.

A qualidade vocal sofre uma modificação; pode haver a presença de tremor ${ }^{(4,10)}$, voz crepitante ${ }^{(9-10)}$, voz rouca, soprosa ou áspera, sendo que esses tipos estariam entre os estereótipos atribuídos a vozes envelhecidas ${ }^{(4,10)}$. Em muitos idosos, nota-se fadiga vocal e estratégias inapropriadas para compensação das mudanças vocais, na tentativa de produzir uma voz melhor, além de instabilidade vocal ${ }^{(4)}$.

Os efeitos do envelhecimento no corpo não são únicos. Muitas vezes, assemelham-se àqueles observados em doenças ou desuso, devido, por exemplo, a repouso ou imobilização prolongada. O desuso leva à perda de fibras do músculo, indistinguíveis daquelas causadas pela idade avançada ${ }^{(11)}$. Os mesmos autores ${ }^{(11)}$ citam que exercícios apropriados evitam ou revertem esses efeitos nos jovens, e parecem ter o mesmo efeito nas mudanças decorrentes do processo de envelhecimento. Esses exercícios, além de manterem o funcionamento e a coordenação muscular, também auxiliam o sistema vascular, nervoso e, especialmente, o respiratório.

Para os mesmos autores ${ }^{(11)}$, a adequada capacidade respiratória está associada à maior eficiência de voz e assim, no caso do envelhecimento vocal, devido ao decréscimo normal da função respiratória com a idade, torna-se essencial um melhor condicionamento não apenas respiratório, mas também físico e da musculatura abdominal.

Dessa forma, a literatura mostra que pessoas idosas, em boas condições de saúde ${ }^{(4,12-13)}$ ou fisicamente ativas ${ }^{(11,14)}$ têm vozes difíceis de serem distinguidas das vozes de falantes jovens. Seguindo essa linha de raciocínio, um treinamento vocal associado a cuidados gerais com a voz ao longo da vida, ou em contrapartida o mau uso da voz ou o abuso vocal também deveriam determinar um efeito no envelhecimento da voz.

Para um melhor entendimento desse aspecto, é importante pensar na voz não apenas como produto de funcionamento orgânico, mas sim, como instrumento de interação social ${ }^{(15)}$, sendo a principal ferramenta de representação dos vários papéis desempenhados no cotidiano, e entre eles o profissional. Este é o caso específico de profissionais da arte (cantores, atores e dubladores); da comunicação (locutores, repórteres e telefonistas); da educação (professores, padres, pastores e fonoaudiólogos) e da área de marketing, indústria e comércio e do judiciário.

Vários estudos na literatura apontam para a incidência de problemas vocais em professores, cuja vozé uma das principais ferramenta de trabalho ${ }^{(16-18)}$, mas não foi encontrada referência específica à voz de professores acima de 65 anos.

As principais alterações vocais verificadas em professores mais jovens mencionadas na literatura referem-se basicamente à qualidade vocal, com prevalência de voz rouca, seguido de aspereza e soprosidade e, foco ressonantal em desequilíbrio $^{(19)}$.

Os distúrbios vocais dos professores limitam seu desempenho e o exercício da profissão; geralmente, pioram ao longo do tempo da atividade profissional ${ }^{(20)}$ e podem interferir na relação com os alunos, dependendo do impacto que lhes causa ${ }^{(16,20)}$.

A princípio poderia se hipotetizar que, se o professor apresenta essa alta incidência de problemas vocais e se, ao envelhecer, há uma tendência para mudanças de voz inerentes ao próprio processo de envelhecimento, o professor idoso, provavelmente deveria carregar as consequiências desse abuso.

Dessa forma, um estudo da voz do idoso associado a uma visão multidimensional do envelhecimento poderia permitir a percepção da heterogeneidade desse processo, de modo a contribuir para a realização de trabalhos na área de prevenção e tratamento dos problemas vocais associados a essa fase da vida.

O presente estudo tem como objetivo comparar os parâmetros vocais de sujeitos idosos professores e não professores e verificar a relação desses parâmetros com a idade cronológica e o tempo de prática de atividade física.

\section{MÉTODOS}

Foi realizada uma pesquisa de caráter observacional e de corte transversal na cidade de São Paulo.

\section{Sujeitos}

Dois grupos de sujeitos participaram dessa pesquisa: o Grupo 1, constituído por sujeitos professores (GP) e o Grupo 2 por não professores (GNP). Como critério de inclusão, para ambos os grupos foram selecionados sujeitos acima de 65 anos, não institucionalizados, homens e mulheres. Para o GP, especificamente, foram incluídos os professores em atividade ou aposentados e, para o GNP, os sujeitos não professores e igualmente não profissionais de voz.

Foram excluídos os sujeitos portadores de doença sistêmica grave, histórico de alcoolismo, consumo de drogas, refluxo gastro-esofágico não tratado, fumantes ou que tenham deixado de fumar há menos de dez anos, com presença de sotaque e que tiveram treinamento de voz anterior.

Todos os sujeitos foram selecionados por meio de um protocolo de entrevista com 26 questões para pesquisa dos critérios de inclusão e exclusão e dados sobre as variáveis idade cronológica e tempo de prática de atividade física. Em relação à atividade física, considerou-se tanto a modalidade aeróbica 
como a musculação, desde que praticadas regularmente, no mínimo, duas vezes por semana e em dias alternados.

\section{Instrumento}

O instrumento utilizado para a análise perceptivo-auditiva do trecho de fala dos sujeitos foi adaptado de protocolo citado na literatura pesquisada ${ }^{(21)}$ e composto por sete parâmetros vocais, a saber, pitch, loudness, ressonância, variações de pitch e de loudness, velocidade e qualidade vocal, pois a literatura indica que esses parâmetros podem estar modificados no envelhecimento. Esses parâmetros foram avaliados em escala de graduação de 1 a 7, conforme Anexo 1.

\section{Procedimentos}

Após a seleção de 47 sujeitos, acima de 65 anos, homens e mulheres, sendo 23 professores (GP) e 24 não professores (GNP), foi coletada uma amostra de fala a partir da reprodução oral de uma história contada pela fonoaudióloga pesquisadora. Essa amostra foi editada em CD digital para a análise dos parâmetros vocais.

A análise perceptivo-auditiva das vozes dos sujeitos do estudo foi realizada no consultório da fonoaudióloga pesquisadora por três fonoaudiólogas, mestrandas do Programa de Estudos Pós Graduados em Fonoaudiologia da Pontifícia Universidade Católica de São Paulo (PUC-SP), com título de especialista em voz e com experiência mínima de três anos. O tempo de experiência foi relevante por se considerar esse o período mínimo para aquisição de experiência na área, uma vez que a análise perceptivo-auditiva refere-se à comparação das vozes ao próprio sistema de referência do avaliador e, portanto, é um critério necessário para não comprometer os resultados da pesquisa.

A quantidade de fonoaudiólogas foi baseada no fato de que o número ímpar favorece o cálculo do nível de confiabilidade das respostas entre os sujeitos. As três fonoaudiólogas-juízes analisaram, ao mesmo tempo, a amostra de fala dos 47 sujeitos, após ouvirem três vezes cada trecho e registraram no protocolo específico os valores correspondentes aos parâmetros propostos para análise.

\section{Análise de dados}

Após a análise da voz de cada sujeito, os parâmetros obtidos pelas três fonoaudiólogas foram analisados quanto à confiabilidade em termos da chamada consistência interna dos valores observados por meio do Teste da Estatística Alfa de Cronbach. Os valores obtidos nessa análise foram estatisticamente elevados (entre 0, 669 e >0,999), e o nível de significância adotado foi $\mathrm{p}<0,001$, o que permitiu considerar a amostra com grau de confiabilidade entre satisfatório e elevado.

Em seguida, para a verificação da reprodutibilidade, foi aplicado o Teste dos Postos Sinalizados de Wilcoxon, para identificação de possíveis diferenças entre os dois momentos (teste e re-teste) de análise das amostras de fala repetidas e randomicamente dispostas no $\mathrm{CD}$ utilizado para essa análise. $\mathrm{O}$ nível de reprodutibilidade foi elevado, pois as diferenças entre os resultados do teste e re-teste não foram estatisticamente significantes, com significância (p) entre 0,317 e 0,917.

Para o prosseguimento da análise foram considerados os valores de cada um dos parâmetros vocais dos sujeitos dos grupos GP e GNP, obtidos em consenso pela maioria. Em seguida, esses dados foram descritos e, pelo fato de não haver um consenso na literatura, os valores correspondentes a cada parâmetro avaliado foram agrupados em três faixas: entre 3,5 e 4,5, significou que o parâmetro avaliado estaria dentro da faixa de normalidade; abaixo de 3,49 ou acima de 4,51, indicou que foi considerado como uma característica que talvez pudesse ser vista como alteração.

Em seguida, os mesmos dados individuais de cada parâmetro vocal (isto é, não agrupados nas três faixas), foram analisados estatisticamente por meio da Análise da Correlação de Spearman para verificação de possíveis diferenças entre os dois grupos de sujeitos. O mesmo teste estatístico foi utilizado ainda para analisar a relação entre os dados da análise perceptivo-auditiva e as variáveis idade cronológica e tempo de prática de atividade física

Todos os sujeitos assinaram o Termo de Consentimento Livre e Esclarecido e esta pesquisa foi aprovada pelo Comitê de Ética do Programa de Estudos Pós-Graduados em Gerontologia da PUC-SP em 01/08/06.

\section{RESULTADOS}

\section{Caracterização dos sujeitos}

Na Tabela 1 está apresentada a caracterização dos sujeitos selecionados na entrevista inicial, quanto à faixa etária, sexo e tempo de prática de atividade física.

Para complementar os dados da Tabela 1, é importante mencionar que, no grupo GP, devido à dificuldade em encontrar-se professores acima de 65 anos de idade em atividade e que tivessem disponibilidade de comparecimento à avaliação, foram selecionados 18 professores universitários $(78,26 \%)$, e cinco professores aposentados $(21,74 \%)$, sendo três de ensino universitário e dois de segundo grau e universitário, mas com menor tempo de dedicação a esse último.

Os professores que ainda estavam atuando, tinham de $20 \mathrm{a}$ 59 anos de docência, (média de 39,1 anos) e com carga horária de quatro a 12 horas por semana (média de 7,1 horas).

\section{Análise perceptivo-auditiva}

Na Tabela 2 está demonstrada a distribuição dos sujeitos GP e GNP em relação a sexo e aos parâmetros vocais.

Na Tabela 3 está demonstrada a distribuição dos sujeitos GP e GNP, em relação a sexo e à qualidade vocal.

Na Tabela 4 está demonstrado o resultado da correlação estatisticamente significante encontrada após o cruzamento das variáveis propostas nesta pesquisa.

Nota-se na Tabela 4 que apenas para os sujeitos GP foi encontrada uma relação estatisticamente significante do parâmetro vocal variação de loudness com a idade cronológica, com correlação negativa e $(\mathrm{p}=0,042)$, de modo que quanto 
Tabela 1. Distribuição numérica e percentual dos sujeitos do GP $(n=23)$ e do GNP $(n=24)$ segundo faixa etária, sexo e tempo de prática de atividade física

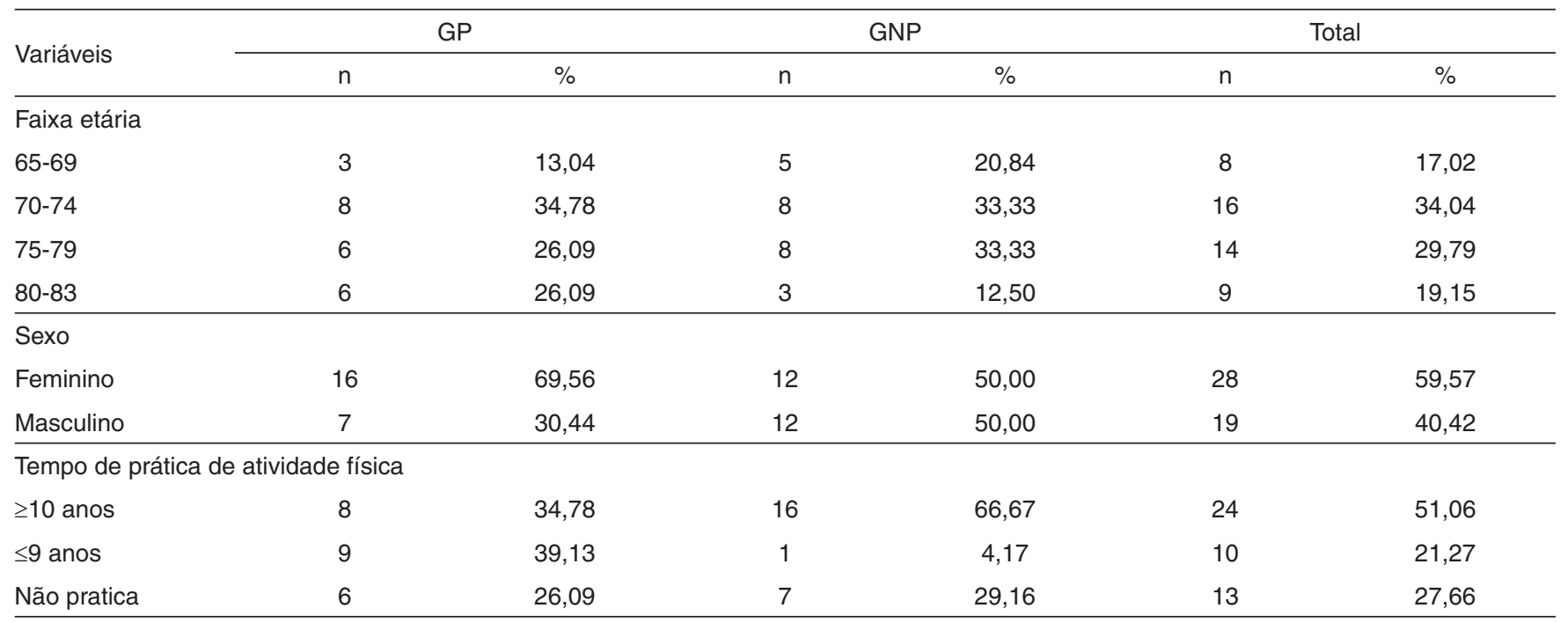

Legenda: GP = grupo de professores; GNP = grupo de não-professores

Tabela 2. Distribuição numérica e percentual dos sujeitos do GP $(n=23)$ e GNP $(n=24)$ em relação ao sexo e aos valores obtidos nos parâmetros vocais (faixa de normalidade: 3,5 a 4,5 )

\begin{tabular}{|c|c|c|c|c|c|c|c|c|c|c|c|}
\hline \multirow{3}{*}{ Parâmetro } & \multirow{3}{*}{ Intervalo } & \multicolumn{4}{|c|}{ GP } & \multicolumn{4}{|c|}{ GNP } & \multirow{2}{*}{\multicolumn{2}{|c|}{ Total }} \\
\hline & & \multicolumn{2}{|c|}{ Masculino } & \multicolumn{2}{|c|}{ Feminino } & \multicolumn{2}{|c|}{ Masculino } & \multicolumn{2}{|c|}{ Feminino } & & \\
\hline & & $n$ & $\%$ & $\mathrm{n}$ & $\%$ & $\mathrm{n}$ & $\%$ & $\mathrm{n}$ & $\%$ & $\mathrm{n}$ & $\%$ \\
\hline \multirow[t]{3}{*}{ Pitch } & $\leq 3,49$ & 3 & 13,04 & 7 & 30,44 & 6 & 25,00 & 7 & 29,16 & 23 & 48,93 \\
\hline & Entre 3,5 e 4,5 & 4 & 17,39 & 6 & 26,09 & 6 & 25,00 & 5 & 20,84 & 21 & 44,68 \\
\hline & $\geq 4,51$ & 0 & --- & 3 & 13,04 & 0 & ---- & 0 & --- & 3 & 6,38 \\
\hline \multirow[t]{3}{*}{ Loudness } & $\leq 3,49$ & 2 & 8,70 & 3 & 13,04 & 2 & 8,33 & 3 & 12,50 & 10 & 21,27 \\
\hline & Entre 3,5 e 4,5 & 4 & 17,39 & 8 & 34,78 & 9 & 37,50 & 4 & 6,67 & 25 & 53,19 \\
\hline & $\geq 4,51$ & 1 & 4,35 & 5 & 21,74 & 1 & 4,16 & 5 & 20,84 & 12 & 25,53 \\
\hline \multirow[t]{3}{*}{ Ressonância } & $\leq 3,49$ & 4 & 17,39 & 5 & 21,74 & 7 & 29,16 & 5 & 20,84 & 21 & 44,68 \\
\hline & Entre 3,5 e 4,5 & 3 & 13,04 & 10 & 43,48 & 5 & 20,84 & 5 & 20,84 & 23 & 48,93 \\
\hline & $\geq 4,51$ & 0 & --- & 1 & 4,35 & 0 & ---- & 2 & 8,33 & 3 & 6,38 \\
\hline \multirow{2}{*}{ Variação de Pitch } & Entre 3,5 e 4,5 & 2 & 8,70 & 9 & 39,13 & 9 & 37,50 & 6 & 25,00 & 26 & 55,31 \\
\hline & $\geq 4,51$ & 4 & 17,39 & 5 & 21,74 & 1 & 4,16 & 6 & 25,00 & 16 & 34,04 \\
\hline \multirow[t]{3}{*}{ Variação de Loudness } & $\leq 3,49$ & 1 & 4,35 & 1 & 4,35 & 2 & 8,33 & 0 & --- & 4 & 8,51 \\
\hline & Entre 3,5 e 4,5 & 3 & 13,04 & 11 & 47,83 & 9 & 37,50 & 8 & 33,34 & 31 & 65,96 \\
\hline & $\geq 4,51$ & 3 & 13,04 & 4 & 17,39 & 1 & 4,16 & 4 & 16,67 & 12 & 25,53 \\
\hline \multirow[t]{3}{*}{ Velocidade } & $\leq 3,49$ & 0 & --- & 0 & --- & 1 & 4,16 & 0 & --- & 1 & 2,13 \\
\hline & Entre 3,5 e 4,5 & 5 & 21,74 & 8 & 34,78 & 10 & 41,67 & 5 & 29,16 & 28 & 59,57 \\
\hline & $\geq 4,51$ & 2 & 8,70 & 8 & 34,78 & 1 & 4,16 & 7 & 29,16 & 18 & 38,30 \\
\hline
\end{tabular}

Legenda: GP = grupo de professores; GNP = grupo de não-professores

maior a idade cronológica, menor a capacidade de variação de loudness.

Por outro lado, observou-se uma relação estatisticamente significante, apenas para os sujeitos não professores (GNP), entre a velocidade e a idade cronológica, com correlação negativa e significância estatística $(\mathrm{p}=0,038)$, que sugere que quanto maior a idade cronológica, menor a velocidade de fala.

Na Tabela 4, percebe-se ainda que apenas para os sujeitos GNP houve uma relação estatisticamente significante entre a qualidade vocal e o tempo de prática de atividade física, com correlação negativa $(\mathrm{p}=0,031)$ e sugere que quanto maior o 
Tabela 3. Distribuição numérica e percentual dos sujeitos do GP $(n=23)$ e do GNP $(n=24)$ em relação a sexo e qualidade vocal

\begin{tabular}{|c|c|c|c|c|c|c|c|c|c|c|}
\hline \multirow{3}{*}{ Qualidade vocal } & \multicolumn{4}{|c|}{ GP } & \multicolumn{4}{|c|}{ GNP } & \multirow{2}{*}{\multicolumn{2}{|c|}{ Total }} \\
\hline & \multicolumn{2}{|c|}{ Masculino } & \multicolumn{2}{|c|}{ Feminino } & \multicolumn{2}{|c|}{ Masculino } & \multicolumn{2}{|c|}{ Feminino } & & \\
\hline & $\mathrm{n}$ & $\%$ & $\mathrm{n}$ & $\%$ & $\mathrm{n}$ & $\%$ & $\mathrm{n}$ & $\%$ & $\mathrm{n}$ & $\%$ \\
\hline Tremor & 0 & --- & 1 & 4,35 & 0 & --- & 1 & 4,16 & 2 & 4,25 \\
\hline Soprosa & 1 & 4,35 & 0 & --- & 1 & 4,16 & 0 & --- & 2 & 4,25 \\
\hline Rouca tremor & 0 & ---- & 2 & 8,70 & 0 & ---- & 0 & ---- & 2 & 4,25 \\
\hline Rouca soprosa & 0 & ---- & 3 & 13,04 & 0 & ---- & 0 & ---- & 3 & 6,38 \\
\hline Rouca & 2 & 8,70 & 4 & 17,39 & 5 & 20,84 & 8 & 33,34 & 19 & 40,42 \\
\hline Gutural & 1 & 4,35 & 0 & --- & 0 & --- & 0 & --- & 1 & 2,13 \\
\hline Hipernasal & 0 & --- & 0 & --- & 1 & 4,16 & 0 & --- & 1 & 2,13 \\
\hline Adequada & 3 & 13,04 & 6 & 26,09 & 5 & 20,84 & 3 & 12,50 & 17 & 34,17 \\
\hline
\end{tabular}

Legenda: GP = grupo de professores; GNP = grupo de não-professores

Tabela 4. Resultado do coeficiente de correlação e da significância (p) obtidos com o cruzamento de variáveis estatisticamente significantes para os grupos de sujeitos do GP $(n=23)$ e do GNP $(n=24)$

\begin{tabular}{lccc}
\hline \multirow{2}{*}{ Par de variáveis } & \multicolumn{2}{c}{ GP } & \\
\cline { 2 - 4 } & Coeficiente de correlação & Significância $(p)$ & Coeficiente de correlação \\
\hline IC x Variação de loudness & $-0,427$ & 0,042 & Significância (p) \\
IC x Velocidade & ------ & $-0,427$ \\
Qualidade vocal x TPAF & ---- & --- & $-0,442$ \\
\hline
\end{tabular}

Legenda: GP = grupo de professores; GNP = grupo de não-professores; IC = idade cronológica; TPAF = tempo de prática de atividade física

tempo de prática de atividade física, menor a ocorrência de desvios na qualidade vocal.

\section{DISCUSSÃO}

Os professores que ainda atuavam encontravam-se na universidade, por ser este o local com maior número de professores idosos em atividade, sugerindo que eles mantinham seus papéis relevantes na sociedade, fato provavelmente associado às boas condições de saúde, com autonomia física e mental, conforme mencionado na literatura ${ }^{(22)}$.

Houve maior porcentagem de sujeitos, professores e não professores, praticantes de algum tipo de atividade física, do que não praticantes, fato que pode demonstrar a conscientização dessa população estudada em relação aos benefícios da atividade física ${ }^{(11)}$.

Os números de sujeitos dos grupos GP e GNP praticantes de atividade física foram semelhantes, com diferença apenas em relação ao maior tempo de inclusão dessa atividade na rotina diária pelos sujeitos GNP.

A descrição dos resultados obtidos pela análise perceptivoauditiva evidencia que, quanto ao pitch, os sujeitos do sexo masculino, tanto do GP como do GNP, não apresentaram pitch mais agudo no trecho de fala avaliado. Essa é uma questão que se apresenta controversa na literatura: alguns autores apontam essa tendência para os homens idosos ${ }^{(4)}$, enquanto outros, por meio de análise acústica (pois não há essa referência em análise perceptivo-auditiva na literatura) afirmam não encontrar essa propensão, independente do sexo e do instrumento de pesquisa utilizado $^{(12,23)}$. A maioria das mulheres dos grupos GP e GNP, obteve resultados indicativos de um agravamento da voz e confirmaram a tendência encontrada na literatura para um abaixamento do pitch para o sexo feminino, provavelmente associado às mudanças hormonais na menopausa ${ }^{(4)}$.

Não há diferença estatisticamente significante quanto ao pitch entre a voz dos sujeitos GP e GNP e nem quanto à idade cronológica ou tempo de prática de atividade física.

Em relação ao loudness, há um predomínio de sujeitos, independente do sexo, tanto do GP como do GNP, com valores correspondentes à faixa de normalidade, fato que poderia confirmar os resultados encontrados na literatura ${ }^{(7)}$, que apesar de modificações fisiológicas inerentes ao envelhecimento, não há mudança na habilidade de conversar em intensidade semelhante à de sujeitos jovens.

Os demais resultados indicaram em ambos os grupos, uma distribuição semelhante de sujeitos tanto com loudness aumentado como diminuído; isto está de acordo com os achados da literatura pesquisada, de que não há um consenso sobre a intensidade vocal do idoso, pois há autor ${ }^{(24)}$ que sugere um aumento de intensidade vocal, não relacionado à presbiacusia enquanto que para outros, a intensidade freqüentemente diminui ${ }^{(5)}$ e portanto haveria uma tendência à redução do loudness ${ }^{(4)}$.

A comparação da voz dos sujeitos GP e GNP quanto ao loudness, mostra que não foi encontrada diferença estatisticamente significante entre os dois grupos, assim como também não há relação estatisticamente significante entre loudness, idade cronológica e tempo de prática de atividade física.

Quanto à ressonância, há um predomínio de sujeitos do GP e do GNP, de ambos os sexos, com ressonância equilibrada (isto é, dentro da faixa de normalidade). A comparação desses 
resultados com a literatura mostra uma diversidade, pois: no caso das mulheres, a literatura indica uma tendência a um foco de ressonância mais baixo ${ }^{(8)}$, fato que nesta pesquisa ocorreu em menor número.

Para os homens idosos, ocorreria o contrário ${ }^{(5)}$, o que não pode ser observado nesta pesquisa. No caso específico do grupo de professores, na literatura consultada ${ }^{(19)}$ verificou-se que a maioria deles, embora mais jovens, apresentam foco ressonantal em desequilíbrio, embora não explicite se o foco era mais anasalado ou laringo faríngeo.

O parâmetro ressonância teve um comportamento estatisticamente semelhante em ambos os grupos e não foi encontrada relação estatisticamente significante entre a ressonância e as variáveis idade cronológica e tempo de prática de atividade física.

Quanto à variação de pitch, independente do sexo, houve um predomínio de sujeitos do GP e do GNP com valores ao redor da normalidade, seguido de sujeitos de ambos os grupos com maior variação de pitch, confirmando, nesse último caso, os resultados citados na literatura sobre um aumento na variabilidade do pitch em sujeitos idosos ${ }^{(5)}$. A diminuição da variação de pitch ocorreu em menor número para ambos os grupos.

Não houve diferença estatisticamente significante quanto à variação de pitch entre os dois grupos e nem relação estatisticamente significante quando comparada com a idade cronológica e tempo de prática de atividade física.

A avaliação da variação de loudness, independente do sexo, mostrou que, para ambos os grupos, há um predomínio de sujeitos que utilizam esse recurso dentro da faixa de normalidade, seguido de, em ordem decrescente de ocorrência, sujeitos com maior variabilidade de loudness e em menor número, sujeitos com variabilidade diminuída.

A diferença da capacidade de utilização desse recurso entre os dois grupos, não foi estatisticamente significante. Apenas para os sujeitos GP foi encontrada uma relação estatisticamente significante, de modo que quanto maior a idade cronológica, menor a capacidade de variação de loudness, fato que poderia ser explicado por um desgaste na voz, provavelmente relacionado ao padrão de voz forte do professor ${ }^{(17,25-26)}$. Tais achados confirmam os dados de literatura $^{(7,9)}$, embora esses autores não tenham feito referência aos sujeitos das respectivas pesquisas como sendo ou não profissionais da voz. O mesmo não pode ser observado no grupo de não professores.

Não foi encontrada relação estatisticamente significante entre a variação de loudness e tempo de prática de atividade física. Os resultados obtidos com a análise da velocidade, independente do sexo, foram semelhantes para os sujeitos GP e GNP, quanto a um predomínio de sujeitos com velocidade normal, seguidos por um número menor de sujeitos com velocidade aumentada. A ocorrência de velocidade diminuída foi em menor número.

Embora não haja diferença estatisticamente significante entre os dois grupos quanto à velocidade de fala, observouse uma relação estatisticamente significante, apenas para os sujeitos não professores (GNP), entre a velocidade e a idade cronológica e sugere que quanto maior a idade cronológica, menor a velocidade de fala; estes dados vão na mesma direção da literatura consultada ${ }^{(10,27)}$ em referência ao envelhecimento vocal de idosos de modo geral, independente da profissão, isto é, se professores ou outros profissionais da voz.

Por outro lado, o aumento da velocidade verificado em sujeitos do GP e do GNP desta pesquisa, poderia estar associado a uma incoordenação pneumofonoarticulatória associada ao envelhecimento que, embora não tenha sido avaliada, pode estar relacionada a uma redução do tempo máximo de fonação e da capacidade respiratória vital ${ }^{(4-5)}$. Assim sendo, os sujeitos poderiam ter aumentado a velocidade com receio de que o ar fosse terminar. Não houve relação estatisticamente significante entre velocidade e tempo de prática de atividade física.

Entre os tipos de qualidade vocal, houve um predomínio de sujeitos nos dois grupos com rouquidão, seguido, em ordem decrescente de ocorrência, de voz adequada, soprosidade e tremor, independente do grau. Apenas para os sujeitos GP percebeu-se uma combinação de rouquidão com soprosidade ou tremor.

A comparação das vozes de sujeitos do GP e do GNP, de modo geral, mostra que não foi encontrada diferença estatisticamente significante na qualidade vocal, independente do sexo. Apesar de que na literatura não foi encontrada referência específica à voz de professores acima de 65 anos, os estudos da qualidade vocal de professores mais jovens apontam para a presença de rouquidão, aspereza e soprosidade ${ }^{(19)}$.

A voz envelhecida também pode apresentar rouquidão, soprosidade, aspereza, tensão, embora o tremor seja mais relacionado à idade avançada ${ }^{(4,9-10,28)}$. A maior ocorrência de tremor para os professores não foi estatisticamente significante, quando comparado com não professores e, portanto, não se pode inferir, para esta amostra, que o tremor estaria mais presente na qualidade vocal de professores envelhecidos do que de idosos não professores.

Não houve relação estatisticamente significante dos resultados da análise da qualidade vocal com a idade cronológica. Apenas para os sujeitos GNP, houve uma relação estatisticamente significante entre a qualidade vocal e o tempo de prática de atividade física, fato que sugere que quanto maior o tempo de prática de atividade física, menor a ocorrência de desvios na qualidade vocal, confirmando os achados de literatura ${ }^{(11,14)}$.

Em resumo, os resultados da avaliação perceptivo-auditiva mostraram que os parâmetros vocais de professores e não professores idosos são semelhantes. Embora não tenha sido encontrado na literatura referência específica a essa comparação, os artigos consultados, sugerem que os parâmetros vocais de idosos, sejam professores ou não profissionais de voz em geral, podem ser semelhantes.

Apesar da relação estatisticamente significante encontrada entre a idade cronológica e dois parâmetros da análise perceptivo-auditiva diferentes (variação de loudness e velocidade), um para cada grupo de sujeitos avaliados (GP e GNP respectivamente), esta não pode ser utilizada como um marcador para o envelhecimento vocal, em concordância com as conclusões da literatura consultada ${ }^{(5,12-13)}$. Esses autores afirmam que a idade cronológica pode ser um critério importante para o agrupamento de sujeitos em uma pesquisa, mas não para a determinação do tipo ou momento de ocorrência de mudanças vocais. 
A dificuldade para se traçar um quadro geral sobre o envelhecimento vocal ${ }^{(5)}$ parece ser o reflexo da própria heterogeneidade do envelhecimento ${ }^{(1,3)}$. Os resultados obtidos reforçam a idéia de que a idade cronológica não pode ser utilizada como um marcador do envelhecimento de forma geral $^{(1-2)}$.

As variáveis idade cronológica e tempo de prática de atividade física e os dois grupos de sujeitos analisados (professores e não professores) forneceram importantes contribuições para um melhor entendimento da heterogeneidade do envelhecimento vocal para que seja possível um trabalho de educação que vise o combate dos mitos e estereótipos associados ao envelhecimento ${ }^{(2,29)}$, o desenvolvimento de programas de prevenção e tratamento de problemas de saúde e bem-estar do idoso, adaptados às diferentes necessidades das muitas velhices ${ }^{(3)}$.

\section{CONCLUSÃO}

Os dois grupos de idosos avaliados (professores e não professores) apresentaram parâmetros vocais semelhantes. A diferença encontrada esteve relacionada às variáveis idade cronológica e tempo de prática de atividade física, de modo que para os professores, quanto maior a idade cronológica, menor a variação de loudness, enquanto que para os não professores, quanto maior a idade cronológica, menor a velocidade de fala. O maior tempo de prática de atividade física relacionou-se a uma qualidade vocal com menos desvios, apenas para os sujeitos não professores.

\begin{abstract}
Purpose: To compare the voices of elderly subjects, teachers and non-teachers, and to verify the relationship between their voices and the variables chronological age and period of time practicing physical activity. Methods: It was carried out the perceptive-auditory analysis of the vocal parameters of 47 subjects, male and female, who were older than 65 years. Twenty three subjects were teachers (GT) and 24 were non-teachers (GNT). The results of this analysis were tested for statistical correlation with the variables chronological age and amount of physical activity practice (in time). Results: In the GT, there was a significant negative correlation between chronological age and loudness variation $(\mathrm{p}=0,042)$. In the GNT it was found significant negative correlations between chronological age and speech rate $(\mathrm{p}=0,038)$ and between period of physical activity practice and voice quality deviations $(\mathrm{p}=0,031)$. Conclusions: There were no statistical differences among the vocal parameters of both groups. For the GP, it was observed that the higher the chronological age, the lesser the loudness variation. For the GNP, the higher the chronological age, the lesser the speech rate. A longer period of physical activity practice was correlated to a voice with lesser quality deviations, only for the GNP.
\end{abstract}

Keywords: Aging; Voice; Aged; Faculty; Motor activity; Exercise; Health of the elderly

\section{REFERÊNCIAS}

1. Markson EW, Hollis-Sawyer LA. Intersections of aging. Readings in social gerontology. Los Angeles: Roxbury Publishing Company; 2000. 512 .

2. Freitas EV, Py L, Neri AL, Cançado FAX, Gorzoni ML, Rocha SM. Tratado de geriatria e gerontologia. Rio de Janeiro: Guanabara Koogan; 2002.

3. Mercadante E. A identidade e a subjetividade do idoso. Rev Kairos. 1998;1(1):59-67.

4. Behlau M, Azevedo R, Pontes P. Conceito de voz normal e classificação das disfonias. In: Behlau M, organizador. Voz: o livro do especialista. Rio de Janeiro: Revinter; c2001, vol.1. p. 54-84.

5. Andrews ML. Adult and geriatric disorders. In: Andrews ML. Manual of voice treatment: pediatrics through geriatrics. 2nd ed. San Diego: Singular Pub. Group; c1999. p. 219-333.

6. Ringel RL, Chodzko-Zajko WJ. Vocal indices of biological age. J Voice. 1987;1(1):31-7.

7. Morris RJ, Brown WS Jr. Age-related voice measures among adult women. J Voice. 1987;1(1):38-43.

8. Linville SE. Acoustic-perceptual studies of aging voice in women. J Voice. 1987;1(1):44-8.

9. Verdonck-de Leeuw IM, Mahieu HF. Vocal aging and the impact on daily life: a longitudinal study. J Voice. 2004;18(2):193-202.

10. Shipp T, Qi Y, Huntley R, Hollien H. Acoustic and temporal correlates of perceived age. J Voice. 1992;6(3):211-6.

11. Sataloff RT, Rosen DC, Hawkshaw M, Spiegel JR. The aging adult voice. J Voice. 1997;11(2):156-60.
12. Ramig LA, Ringel RL. Effects of physiological aging on selected acoustic characteristics of voice. J Speech Hear Res. 1983;26(1):2230 .

13. Ramig LO, Gray S, Baker K, Corbin-Lewis K, Buder E, Luschei E, et al. The aging voice: a review, treatment data and familial and genetic perspectives. Folia Phoniatr Logop. 2001;53(5):252-65.

14. Xue A, Mueller PB. Acoustic and perceptual characteristics of the voices of sedentary and physically active elderly speakers. Log Phon Vocol. 1997;22(5):51-60.

15. Ferreira LP. Uma pesquisa, uma proposta, um livro: três histórias que se cruzaram. In: Ferreira LP, Oliveira IB, Quinteiro EA, Morato EM, organizadores. Voz profissional: o profissional da voz. Carapicuíba: Pró Fono; 1995. p. 1-6.

16. Dragone ML. Voz do professor: interfaces e valor como instrumento de trabalho. [dissertação]. Araraquara: Universidade Estadual de São Paulo - UNESP; 2000.

17. Ferreira LP, Giannini SPP, Figueira S, Silva EE, Karmann DF, Souza TMT. Condições de produção vocal de professores da Prefeitura do Município de São Paulo. Disturb Comun. 2003;14(2):275-307.

18. Delcor NS, Araújo TM, Reis EJFB, Porto LA, Carvalho FM, Silva MO, et al. Condições de trabalho e saúde dos professores da rede particular de ensino de Vitória da Conquista, Bahia, Brasil. Cad Saude Publica = Rep Public Health. 2004;20(1):187-96.

19. Oliveira IB. Desempenho vocal do professor: avaliação multidimensional. [Tese de Doutorado]. Campinas: Pontifícia Universidade Católica de Campinas; 1999. 
20. Dragone MLS, Sichirolli S, Reis R, Behlau M. O desgaste vocal do professor: um estudo longitudinal. Rev Soc Bras Fonoaudiol. 1999; 3(5):50-6.

21. Arruda AF. Expressividade oral de professores: análise dos recursos vocais. [dissertação ]. São Paulo: Pontifícia Universidade Católica de São Paulo; 2004.

22. Giatti L, Barreto SM. Saúde, trabalho e envelhecimento no Brasil. Cad Saude Publica = Rep Public Health. 2003;19(3):759-71.

23. Hollien H, Dew D, Philips P. Phonational frequency ranges of adults. J Speech Hear Res. 1971;14(4):755-60.

24. Hollien H. "Old voices": what do we really know about them? J Voice. 1987;1(1):2-17.
25. Oyarzun R, Brunetto B, Mella L, Avila S. Disfonia en profesores. Rev Otorrinolaringol Cir Cabeza Cuello. 1984;44(2):12-8.

26. Roy N. Teachers with voice disorders. Recent clinical trials research. The ASHA Leader. 2005;10(5):10-1.

27. Ryan WJ. Acoustic aspects of the aging voice. J Gerontol. 1972;27(2):265-8.

28. Alarcos AL, Behlau M, Tosi O. Computer acoustical analysis of senile voices. Folia Phoniatr. 1983;35:102.

29. Ory M, Kinney Hoffman M, Hawkins M, Sanner B, Mockenhaupt R. Challenging aging stereotypes: strategies for creating a more active society. Am J Prev Med. 2003;25(3 Suppl 2):164-71.

Anexo 1. Protocolo de análise perceptivo-auditiva

Sujeito $n^{\circ}$

Data:

Parâmetro

Pitch

$\begin{array}{ccc}\mid & \mid & \\ 1 & 4 & 7 \\ \text { muito grave } & \text { adequado } & \text { muito agudo }\end{array}$

Loudness

$\begin{array}{ccc}\mid & \mid & \mid \\ 1 & 4 & 7 \\ \text { diminuído } & \text { adequado } & \text { aumentado }\end{array}$

Ressonância

$\begin{array}{ccc}\mid & \mid & \mid \\ 1 & 4 & 7 \\ \text { laríngea } & \text { equilibrada } & \text { nasal }\end{array}$

Variação

Pitch

\begin{tabular}{ccc}
\hline & & \\
1 & 4 & 7 \\
restrita & & muito variada
\end{tabular}

Variação

Loudness

\begin{tabular}{ccc}
\hline & & \\
1 & 4 & 7 \\
restrita & & muito variada
\end{tabular}

Velocidade

$\begin{array}{ccc}\mid & & \\ 1 & 4 & 7 \\ \text { muito lenta } & & \text { muito rápida }\end{array}$

Qualidade Vocal: 